\title{
Investigating Los Angeles' urban roadway network from a biologically-formed perspective
}

\author{
Sophia Deen ${ }^{1}$, Tatiana Kuzmenko ${ }^{2}$, Hossein Asghari ${ }^{3}$, Demian A Willette ${ }^{\text {Corresp. } 2}$ \\ ${ }^{1}$ Department of Health and Human Sciences, Loyola Marymount University, Los Angeles, CA, United States \\ 2 Biology Department, Loyola Marymount University, Los Angeles, CA, United States \\ 3 Department of Electrical Engineering and Computer Science, Loyola Marymount University, Los Angeles, CA, United States \\ Corresponding Author: Demian A Willette \\ Email address: demian.willette@Imu.edu
}

The evolution of networks is constrained by spatial properties of the environment; a characterization that is true in both biological and built networks. Hence built networks such as urban streets can be compared to biological networks to reveal differences in efficiency and complexity. This study assessed foraging networks created by the slimemold Physarium polycephalum on proportional 3D-printed topographic maps of metropolitan city of Los Angeles, California. Rapidly-generated isomorphic solutions were found to be consistently and statistically shorter than existing roadways in system length. Slime mold also allocated resources to supporting key nodes, analogous to how heavy traffic flows through major intersections. Further, chemical deterrents inhibited exploration of slime mold in selected areas and allows for testing of network redundancy and system resilience, such as after an earthquake or wildfire. 


\section{Investigating Los Angeles' urban roadway network from a biologically-formed perspective}

2

3 Sophia H. Deen ${ }^{1}$, Tatiana Kuzmenko², Hossein Asghari ${ }^{3}$, Demian A. Willette ${ }^{2}$

4

$5{ }^{1}$ Department of Health and Human Sciences, Loyola Marymount University, Los Angeles, CA, $6 \quad$ United States

$7 \quad 2$ Biology Department Loyola Marymount University, Los Angeles, CA, United States

$8{ }^{3}$ Department of Electrical Engineering and Computer Science, Loyola Marymount University, 9 Los Angeles, CA, United States

10 Corresponding Author:

11 Demian Willette

121 LMU Drive, Los Angeles, CA 90045, United States

13 Email address: demian.willette@1mu.edu 


\section{Abstract}

15 The evolution of networks is constrained by spatial properties of the environment; a

16 characterization that is true in both biological and built networks. Hence built networks such as urban streets can be compared to biological networks to reveal differences in efficiency and complexity. This study assessed foraging networks created by the slime-mold Physarium polycephalum on proportional 3D-printed topographic maps of metropolitan city of Los Angeles, California. Rapidly-generated isomorphic solutions were found to be consistently and statistically shorter than existing roadways in system length. Slime mold also allocated resources to supporting key nodes, analogous to how heavy traffic flows through major intersections. Further, chemical deterrents inhibited exploration of slime mold in selected areas and allows for testing of network redundancy and system resilience, such as after an earthquake or wildfire.

\section{Introduction}

Networks are created where paths crisscross one another, forming a collection of nodes in space (Barthelemy 2011). Networks are commonly found across biological and built environments to facilitate transport of materials and information. Cardiovascular systems, neural networks, ant tunnels and termite colonies are all examples of biological transport networks, whereas roadways, power grids, sewage systems, and telecommunications are built infrastructure examples. The evolution of a network and resulting topography are both constrained by spatial properties of the environment; a characterization that is true in both biological and built networks. Hence built networks such as urban streets can be compared to biological networks to reveal differences in efficiency and complexity (Jiang et al. 2008). Urban road networks are essential for the movement of goods, people, and services in metropolitan areas, yet these networks were built over many years that span dynamic economic, political, technological and legal periods that did not inherently promote maximum transit efficiency. Economic drivers may discourage redundancy, yet the availability of multiple pathways allows for the self-healing of networks (Quattrociocchi et al. 2014) and ensures network resilience after disruptions (Tero et al. 2010).

The ways in which urban road networks are constructed has been examined in an assortment of perspectives including through statistics (Samaniego and Moses 2008) and computational modeling ( $\mathrm{Li}$ et al. 2015). Roadway creating models typically involve advanced mathematical models that may be difficult to interpret (Yang et al., 1998), which may impede many from truly understanding computed designs, including stakeholders who would benefit from a greater understand of network formation. Even when artfully sketched out designs of proposed networks are created, there are limitations in how closely these models may be compared to real-world networks (Jiang 2009). The mathematically designed models can be a crucial first look at roadways particularly for their accuracy and clarity yet having a more tangible and physical model would be beneficial in other ways such as seeing how the network would interact in its environmental surroundings (Samaniego and Moses 2008). Furthermore, translating urban 
54 roadway models created mathematically from two-dimensions to three-dimensions are

55

56

57

58

59

60

61

62

63

64

65

66

67

68

69

70

71

72

73

74

75

76

77

78

79

80

81

82

83

84

85

86

87

88

89

90

91

92

93

traditionally costly and time consuming to construct, yet provide valuable insight for comparison with real-world networks. A complementary approach to exploring built infrastructure systems is by using biologically created networks, specifically the network-constructing plasmodial slime mold Physarium polycephalum (Tero et al. 2010, Adamatzky and Akl 2011, Adamatzky 2014).

Physarum polycephalum is an aceullar, multinucleated ameboid organism that can be observed with the naked eye. In its vegetative stage called plasmodium, $P$. polycephalum explores the environment in search for food (decaying organic matter) using chemotaxis and a contiguous distribution strategy to maximize the area covered. The plasmodium can explore its terrain at a rate up to 5 centimeter per hour (Kessler et al. 1982). Once food is identified, the plasmodium connects to the food by developing a complex and highly optimized network of pulsating veins or tubes. Plasmodium tubes contain actin and myosin as the components to achieve locomotion, shuttle streaming, and transportation of nutrients and chemical signals within the organism (Alt 1985). It has been shown repeatedly that Physarium exhibits intelligent-like decision making and algebra computing abilities while maintaining the plasmodial network (Nakagaki et al. 2001, Tero et al. 2010, Evangelidis et al. 2017). For example, the plasmodial slime mold has the capability of creating networks isomorphic to that of the Tokyo Railway system, which is highly regarded for its efficiency (Nakagaki et al. 2001, Tero et al. 2010). This is especially impressive given the plasmodial network develops within a few days without any centralized control, whereas the railroad network required years of design and formation effort from civil engineers. What has been lacking in the majority of studies (Adamatzky and Jones 2010, Tero et al. 2010, Adamatzky 2014, Adamatzky et al. 2014) on this plasmodium is that they were conducted on the flat surface in the absence of real-world topographic features such as mountains and valleys that have a dramatic impact on the development of the road networks (but see Adamatzky 2014, Evangelids et al. 2017 as exceptions). Furthermore, slime mold is gravisensitive and positively geotrophic (Moore and Cogoli 1996) meaning in a 3D environment, it will avoid higher elevations and adjust its network structure to accommodate the landscape (Evangelids et al. 2017). This biological feature and an increased accessibility of three-dimensional (3D) printing technology makes it possible to investigate the potentially powerful capacity of slime mold as a tool for exploring and potentially re-envisioning urban roadway networks in the cities where prominent geographical features have a strong impact on transportations network development.

Here we examine how the biological network-building $P$. polycephalum explores the major metropolitan city of Los Angeles, California and compare it to the existing system of freeways. Our study focuses on the downtown Los Angeles region, an area nestled between mountains and foothills, flat basin, and the Los Angeles River, spanning an elevation of 50-500 meters above sea level. The urban roadways and associated traffic patterns of Los Angeles have been the focus of study for decades (Stonex 1957, Teague et al. 1972, Boarnet et al. 1998) and continue to encourage novel thinking in solving congestion and reducing commute times (Wang et al. 2018,

Peer] reviewing PDF | (2019:07:39502:2:0:NEW 5 Nov 2019) 
94 Zhou et al. 2018, Gonzalez 2019). As the United States' second largest city and among the most

95

96

97

98

99

100

101

102

103

104

105

106

107

108

109

110

111

112

113

114

115

116

117

118

119

120

121

122

123

124

125

126

127

128

129

130

131

132

133

densely populated (US Census Bureau 2015), Los Angeles is widely known for its extensive roadways and congested traffic. Seemingly paradoxically, the combination of Los Angeles' spatial extent and longer road segments contribute to its ranking as the top U.S. city for accessibility to jobs by automobile (Levinson 2012), with higher job accessibility statistically correlated with shorter commute times in the city (Hu 2015).

This study specifically aims to assess the exploration of the slime mold's foraging on proportional geographic locations of 3D printed topographical maps of Los Angeles. The results from $P$. polycephalum's exploration will be used to assess the slime mold's ability to generate isomorphic solutions similar to Los Angeles' existing roadway. Slime mold and real-world roadway lengths will be calculated and contrasted to identify which are shorter, thus identify a potentially more efficient network system. This study is not the first to attempt to combine emerging 3D printing technology with network-solving capability of $P$. polycephalum (see Evangelids et al. 2017), yet to our knowledge, it is the first to couple the two to examine applied network design at the scale of a single metropolitan city.

\section{Materials \& Methods}

Proportional topographic maps of a $12 \mathrm{~km}$ by $15 \mathrm{~km}$ area of downtown Los Angeles were captured from Google Earth (Alphabet, California), edited using the Blender (Roosendall, 1998) and MakerBot (Stratasys, New York) software packages and converted into stereolithography files (.stl) using .stl generator software, and printed on a Makerbot $1^{\text {st }}$ Generation Replicator. To have a measurable effect of plasmodium behavior all the elevation values were enhanced 2.5 times. $12 \mathrm{~cm} \times 15 \mathrm{~cm}$ printed maps (1:100,000 scale) were marked with a dot of permanent paint in fixed locations where the food source (oat flakes) could be placed identically through replicated trials. Seven food source locations were chosen intentionally to align with major intersections of both highways and major roadways in Downtown Los Angeles.

For each trial each 3D topographic map was placed in a large $20 \times 20 \mathrm{~cm}$ square petri dish containing an absorbent towel soaked in deionized water. The map, dish, and towel were sanitized for one hour under a UV light to inhibit any fungal growth or contamination. After sanitization, a thin coat of autoclaved non-nutritive $2 \%$ agar was painted over the surface of the 3D maps. The agar media provided a superior growing surface to bare plastic yet was sufficiently thin enough to not distort scaling of the 3D maps. Agar was allowed to solidify and was then ready for the placement of slime mold and oat flakes at fixed starting locations.

Plasmodial P. polycephalum was cultured into its active form from purchased dry sclerotium (Carolina Biological Supply Company, North Carolina), slime mold's dormant stage. The dry sclerotium was placed in the center of a Petri dish filled with solidified, non-nutritive $2 \%$ agar and several oat flakes. The sclerotium was inoculated with deionized water and colonize UV- 
134 sterilized oat flakes in a dark and humid $25^{\circ} \mathrm{C}$ incubator for 48 hours. Three oat flakes inoculated

135

136

137

138

139

140

141

142

143

144

145

146

147

148

149

150

151

152

153

154

155

156

157

158

159

160

161

162

163

164

165

166

167

168

169

170

171

172

173

with plasmodial slime mold and four additional sterile oat flakes were placed on fixed locations of the map. The slime mold was incubated at $25^{\circ} \mathrm{C}$ with no light for 72 hours in a custom-built incubator to allow $P$. polycephalum to fully explore the map and create its network. Preliminary trials revealed 72 hours to be ample time for full exploration of the 3D map by the slime mold. The need for the custom-built incubator came from the objective to take photographs of the entire map at 6-hour intervals over the period of each trial, but without exposing the maps to potential airborne fungal contaminants or to extended periods of light which both alter slime mold growth and behavior. In collaboration with two electrical engineering students at Loyola Marymount University, the incubator was built from off-the-shelf plastic bin and fitted with a Raspberry Pi camera and Graphical User Interface that enabled with a Python Script and an Optical Coherence Tomography System to capture photos of exploration of the slime mold over the map. Full description of the Slime Mold Incubator Camera System (SMICS) is detailed in McGrath et al. 2018 (Figure 1). From the maps generated at the end of the 72-hour exploration period, distances between oat flakes were measured using ImageJ software, and statistical analyses were conducted to compare the plasmodial slime mold's exploration networks with the analogous real-world urban roadway network in downtown Los Angeles (Figure 2).

\section{Results}

Seventeen trials of the plasmodial slime mold's exploration on the proportional 3D topographical maps of the same region of Downtown Los Angeles were conducted (Figure 3). The placement of oat flakes varied only slightly between the study's 17 replicates; however, the difference was not statistically significant (One Way ANOVA, $\mathrm{F}=0.04, \mathrm{DF}=2, \alpha=0.05, p=0.96$ ). The slime mold's overall exploration on the 3D maps of downtown Los Angeles was found to be shorter than human engineered, pre-existing roadway networks (Figure 4). Slime mold networks were on $65.6 \mathrm{~km} \pm 13.8 \mathrm{~km}$ (Average \pm Standard Error) of equivalent length shorter than downtown Los Angeles' current urban roadway network. Furthermore, the number of oat flakes explored in each of the maps was suggested to positively affect the efficiency the slime mold had to explore larger distances. Statistical comparisons of the distances between pairs of real-world intersections and matching pairs of oat flakes revealed a significantly shorter pathway was created by the slime mold than that of existing roadways ( $\mathrm{t}$-test for two independent groups $\mathrm{t}=$ $5.37, \mathrm{DF}=218, \alpha=0.05, \mathrm{p}<0.0001)$.

\section{Discussion}

This study confirms that the plasmodial slime mold $P$. polycephalum can create networks comparable to real-world built networks as described in past 2D map studies (Nakagaki et al. 2001, Tero et al. 2010). More so, this study illustrates that when presented with 3D printed maps, specifically those at the city-scale with real-world topographic features, the slime mold identifies pathways that are typically shorter and thus potentially more cost-effective than pre-existing city roadway networks. This is valuable because explorations such as these can reveal not only 
174 shorter pathways, but also variables such as network connectivity that if even slightly improved

175

176

177

178

179

180

181

182

183

184

185

186

187

188

189

190

191

192

193

194

195

196

197

198

199

200

201

202

203

204

205

206

207

208

209

210

211

212

213

can reduce traffic commute time (Levinson 2012).

The connectivity of the network provides insight into how paths are organized hierarchically. Jiang (2009) determined that a minority of roadways (20\%) handle the majority of traffic ( $80 \%)$, with the top $1 \%$ of paths managing $20 \%$ of traffic flow. Thus, although the current study focused on a limited number of fixed locations (i.e. a fixed number of oat flake food sources) in the construction of the network, these locations were where major highways and roadways intersect and heavy traffic flows. Importantly, there is a cost associated with the lengths within a network that must span a large area and these lengths can dramatic influence the overall topography of the network (Barthelemy 2011). In an urban area, a single road length may be necessary to connect an isolated neighborhood or industrial facility to the rest of the network, consequently adding to the overall expansion and associated maintenance cost of the roadway network. A limitation of our experimental design was our focus on major roadway intersections and future work may examine such isolated, yet important elements of an urban landscape at a higher resolution, a task shown possible here with our examination using a scaled 3D map of only a $12 \mathrm{~km} \times 15 \mathrm{~km}$ area of downtown Los Angeles.

Observed shorter slime mold pathways than real-world roadways may be influenced by how each network was created. Urban roadways are built over time, often in a non-linear progression with inherent restrictions including land ownership, economic resources, and a wide range of social, political, and historical considerations. Furthermore, the development of real-world roadways may be modeled by preferential attachment (Barabasi and Albert 1999) - the concept that new nodes prefer to link to already well-connected network nodes. In an urban scenario, a new housing or commercial development may preferentially be connected directly to a population center, but at a net cost to the overall efficiency of the roadway network. In contrast, slime mold's exploration follows a minimalist strategy creating the most efficient links while curtailing resource expenditure (Adamatzky and de Oliveira 2011). Slime mold will abandon explored pathways that are no longer the most efficient for its metabolic function (achieved by leaving a non-living mat of transparent extracellular slime) (Reid et al. 2012), whereas urban roadways are not typically decommissioned or removed.

A high level of network similarity was qualitatively identified among the 17 72-hour iterations. These data suggest there are certain pathways across the landscape that are important to the overall connectivity and efficiency of the network. Recognizing which routes are important allows for resilience testing of the network in the event of a failure (Quattrociocchi et al. 2014), such as in an earthquake, landslide, or anthropogenic disaster. Future studies may playout such scenarios by applying chemical deterrents such as sodium chloride (Adamatzky and de Oliveira 2011) or a light beam (Tero et al 2010) to inhibit the exploration of slime mold into disturbed areas, and then identifying the most efficient alternative routes.

Peer) reviewing PDF | (2019:07:39502:2:0:NEW 5 Nov 2019) 
214

215 Urbanization will continue in the coming decades with $68 \%$ of the world's population predicted

216 to live in cities (United Nations 2018). Urban growth often facilitates economic development

217 but is also typically associated with the undesirable effects of increased traffic congestion and 218 pollution (Quaas and Smulders 2018). The application of emerging, yet appropriate technology

219 may counter these unintended outcomes. For example, although between 1980 and 2000 the 220 greater Los Angeles area grew by more than $40 \%$ and total vehicle mileage increased by $88 \%$, 221 ambient air quality including ozone, sulfur dioxide, carbon monoxide and lead all significantly 222 improved with the application of vehicle emission reducing technology (Kahn and Schwartz 223 2008). Likewise, we demonstrate here the potential for 3D printing technology paired with 224 225 226

227

228

229

230

231

232

233

234

235

236

237

238

239

240

241

242

243

244

245

246

247

248

249

250

251 plasmodial slime mold can inform and guide network construction of urban roadways and potentially other infrastructure systems across real-world geographic features.

\section{Conclusions}

Our data indicate slime mold presented with real-world topographic features can identify pathways shorter than current existing roadway networks, and the reoccurrence of certain links in multiple test iterations identify where key routes are in the urban roadway network. Given the multiple costs and benefits of urban roadway networks and how increased efficiency of networks can amplify the benefits, we argue for broader inclusion of biologically-inspired design, including the above described inexpensive and rapid approach of coupling slime mold and 3D print technology, in urban roadway discussions and planning.

\section{Acknowledgements}

The authors thank Daniel McGrath and Stephen Board of the LMU Department of Electrical Engineering and Computer Science for the tremendous contribution in developing the SCIMS system for photo-documenting the growth and behavior of the slime mold. Thanks also to Dr. Michelle Yeung for technical support in printing the 3D maps, and the LMU Summer Undergraduate Research Program for early support of S.D. D.W. thanks Dr. Marga Joaquin for logistical support.

\section{References}

Adamatzky, A.I. and J. Jones. 2010. Road planning with slime mould: if Physarum built motorways it would route M6/M74 through Newcastle. International Journal of Bifurcation and Chaos, 20:3065-3084.

Adamatzky, A.I. and S.G. Akl. 2011. Trans-Canada slimeways: Slime mould imitates the Canadian transport network. arXiv preprint arXiv:1105.5084.

Adamatzky, A.I. and P.P.B. de Oliveira. 2011. Brazilian highways from slime mold's point of view. Kybernetes, 40:1373-1394. 
252

253

254

255

256

257

258

259

260

261

262

263

264

265

266

267

268

269

270

271

272

273

274

275

276

277

278

279

280

281

282

283

284

285

286

287

288

289

290

291

Adamatzky, A.I. 2014. Route 20, autobahn 7, and slime mold: approximating the longest roads in USA and Germany with slime mold on 3-D terrains., IEEE Transactions on Cybernetics, 44:126-136.

Adamatzky A.I., R. Armstrong, B.D.L. Constello, Y. Deng, J. Jones, R. Mayne, T. Schubert, X. Zhang, and G.C. Sirakoulis. 2014. Slime mould analog models of space exploration and plant colonization. Journal of the British Interplanetary Society, 67:290-304.

Alt, W. 1985. Contraction and oscillation in a simple model for cell plasma motion. In Temporal Order, Springer, Berlin, 163-174.

Barabasi, A.L. and R. Albert. 1999. Emergence of scaling in random networks. Science, 286:509-512.

Barthelemy, M. 2011. Spatial Networks. Physics Reports 499:1-101.

Boarnet, M.G., E.J. Kim, and E. Parkany. 1998. Measuring traffic congestion. Transportation Research Records 1634:93-99.

Evangelids, V., J. Jones, N. Dourvas, M.A. Tsompanas, G.C. Sirakoulis, and A.I. Adamatzky. 2017. Physarium machines imitating a Roman road network: the 3D approach. Scientific Reports 7:7010.

Gonzalez, D. 2019. Traffic congestion in California: Implementation of congestion pricing in Los Angeles County as an effective traffic-reducing strategy. Doctoral dissertation, California State University, Northridge.

$\mathrm{Hu}, \mathrm{L}$. 2015. Changing effects of job accessibility on employment and commute: A case study of Los Angeles. The Professional Geographer 67:154-165.

Jiang, B., S. Zhao, and J. Yin. 2008. Self-organizing natural roads for predicting traffic flow: a sensitivity study. Journal of Statistical Mechanics: Theory and experiment P07008.

Jiang, B. 2009. Street hierarchies: A minority of streets account for a majority of traffic flow. International Journal of Geographical Information Science 23:1033-1048.

Kahn, M.E., and J. Schwartz. 2008. Urban air pollution progress despite sprawl: the 'greening' of the vehicle fleet. Journal of Urban Economics 63: 775-787.

Kessler, D., H.C. Aldrich, and J.W. Daniel. 1982. Plasmodial structure and motility. Cell Biology of Physarium and Didymium. Academic Press, Sydney, Australia.

Levinson, D. 2012. Network structure and city size. PLoS one 7:e29721.

Li, D. B. Fu, Y. Wang, G. Lu, Y. Berezin, H.E. Stanley, and S. Havlin. 2015. Percolation transition in dynamical traffic network with evolving critical bottlenecks. Proceedings of the National Academy of Sciences 112:669-672.

McGrath, D., S. Board, S. Deen, T. Kuzmenko, D.A. Willette, and H. Asghari. 2018. Physarium polycephalum growth monitoring and optical coherence tomography. LMU Attic Salt publication, Los Angeles, California.

Moore, D. and Cogoli, A., 1996. Gravitational and space biology at the cellular level. In Biological and medical research in space (pp. 1-106). Springer, Berlin, Heidelberg.

Nakagaki, T. H. Yamada, and A. Toth. 2001. Path finding by tube morphogenesis in an amoeboid organism. Biophysical Chemistry 92:47-52. 
292 Quaas, M.F., and S. Smulders. 2018. Brown growth, green growth, and the efficiency of 293 urbanization. Environmental Resource Economics 71: 529-549.

294 Quattrociocchi, W., G. Caldarelli, and A. Scala. 2014. Self-healing networks: redundancy and 295 structure. PLoS one 9:e87986.

296 Reid, C.R., T. Latty, A. Dussutour, and M. Beekman. 2012. Slime mold uses an externalized 297 spatial 'memory' to navigate in complex environments. Proceedings of the National 298 Academy of Sciences, 109:17490-17494.

299 Samaniego, H. and M.E. Moses. 2008. Cities as organisms: Allometric scaling of urban road 300 networks. Journal of Transport and Land Use 1:21-39.

301 Stonex, K.A. 1957. Survey of Los Angeles traffic characteristics. In Highway Research Board $302 \quad$ Proceedings vol 36.

303 Teague, D.M., W. Bishop, L.H. Nagler, G. Onishi, M.V. Sink, K.A. Stonex, and R.T. 304 VanDerveer. 1972. Los Angeles traffic pattern survey. SAE Program and Technology, $305 \quad$ United States 6.

306 Tero, A., S. Takagi, T. Saigusa, K. Ito, D.P. Bebber, M.D. Fricker, and T. Nakagaki. 2010. Rules 307 for biologically inspired adaptive network design. Science 327:439-442.

308 United Nations. 2018. World Urbanization Prospects: the 2018 Revision. 309 https://population.un.org/wup/Publications/Files/WUP2018-KeyFacts.pdf

310 Wang, H. M. Mazari, M. Pourhomayoun, J. Smith, H. Owens, and W. Chernicoff. 2018. An end311 to-end traffic vision and counting system using computer vision and machine learning:

312 The challenges in real-time processing. SIGNAL Editors. P.13.

313 Yang H., and M.G. Bell. 2009. Food quality affects search strategy in the acellular slime mould 314 Physarium polycephalum. Behavioral Ecology 20:1160-1167.

315 Zhou, J., E. Murphy, and J. Corcoran. 2018. Integrating road carrying capacity and traffic 316 congestion into the excess commuting framework: The case of Los Angeles. Environment and Planning B: Urban Analytics and City Science p.2399808318773762. 
Figure 1

Photograph of custom-built Slime Mold Incubator Camera System

Photograph of the custom-designed and built Slime Mold Incubator Camera System (SMICS) used to capture the exploration of the slime mold over the 3D printed maps with one of the $12 \mathrm{~cm} \times 15 \mathrm{~cm}$ maps included for scale.

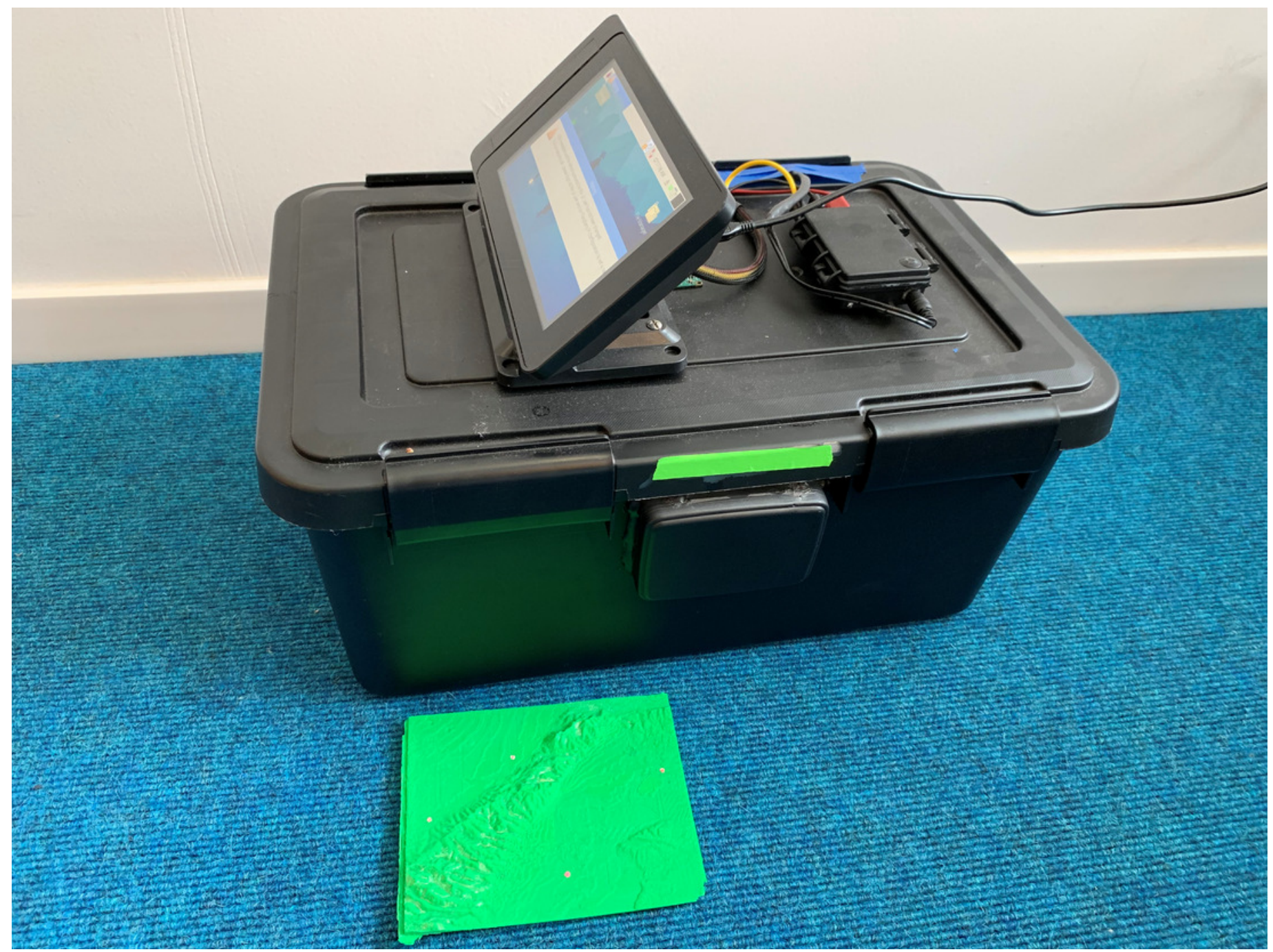




\section{Figure 2}

Slime mold data generation sequence from real-world map to processed data.

Slime mold data generation sequence including (A) real-world aerial map with selected intersections of major roadways (red circles), (B) printed 3D map with topographic features and fixed locations (black dots), (C) representative 3D map with inoculated oat flakes at fixed locations, (D) resulting slime mold network (yellow threads) after 3-day incubation, (E) ImageJ software visualizing network connections, and (F) example length measurement of both freeform and direct distance between nodes using Imagej tool.

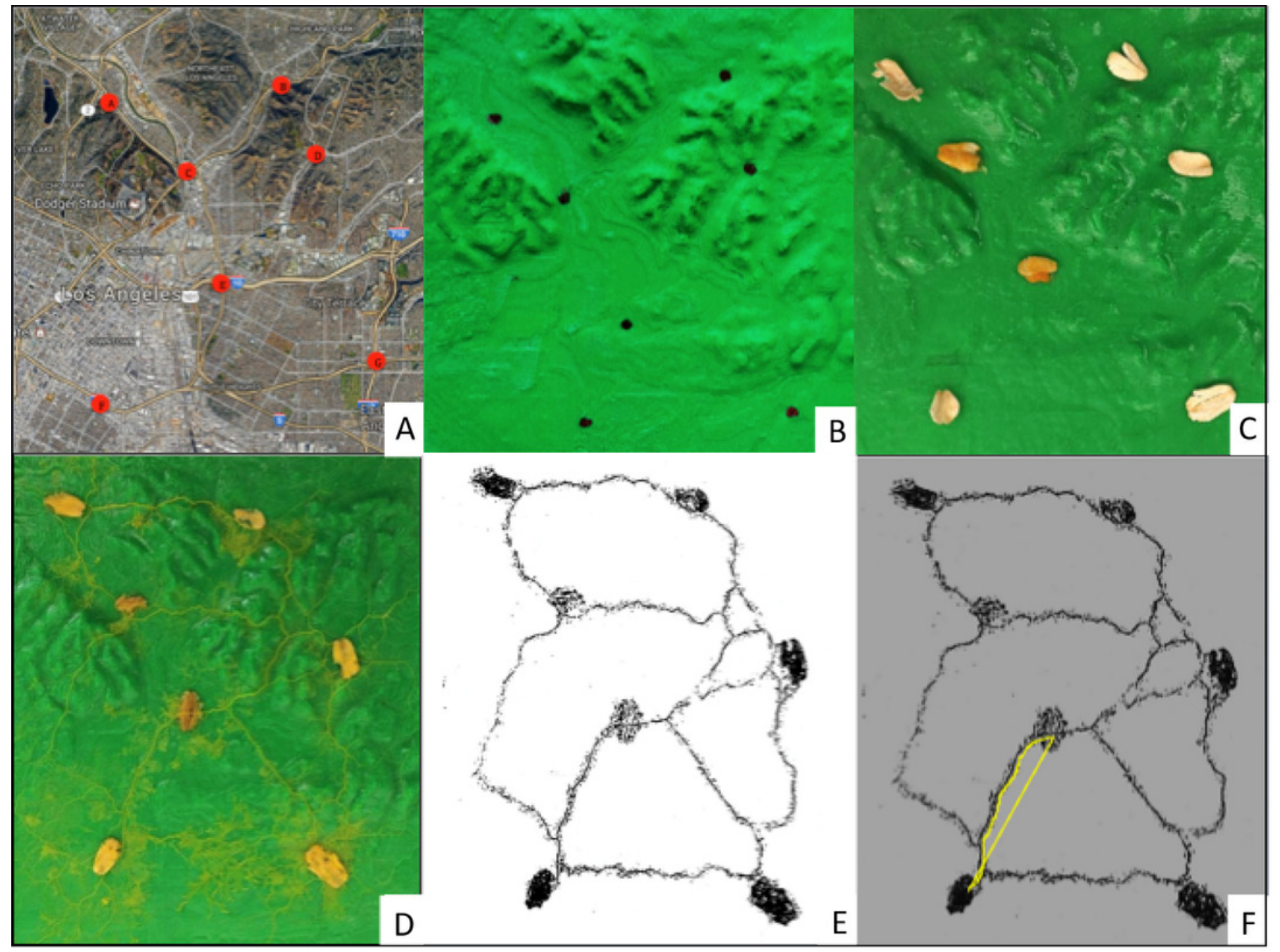




\section{Figure 3}

Comparison of slime mold exploration and existing road network

Visual representation comparing the networks of the existing highway and road networks (A) to those that were created by the slime mold's exploration (B). This image also depicts the locations of the nodes of where the food sources (oat flakes) were placed on the 3D printed maps of Downtown Los Angeles. Image area approximately $12 \mathrm{~km}$ by $15 \mathrm{~km}$. Map data $\odot$ 2018 Google .

Real World Highways and Roads

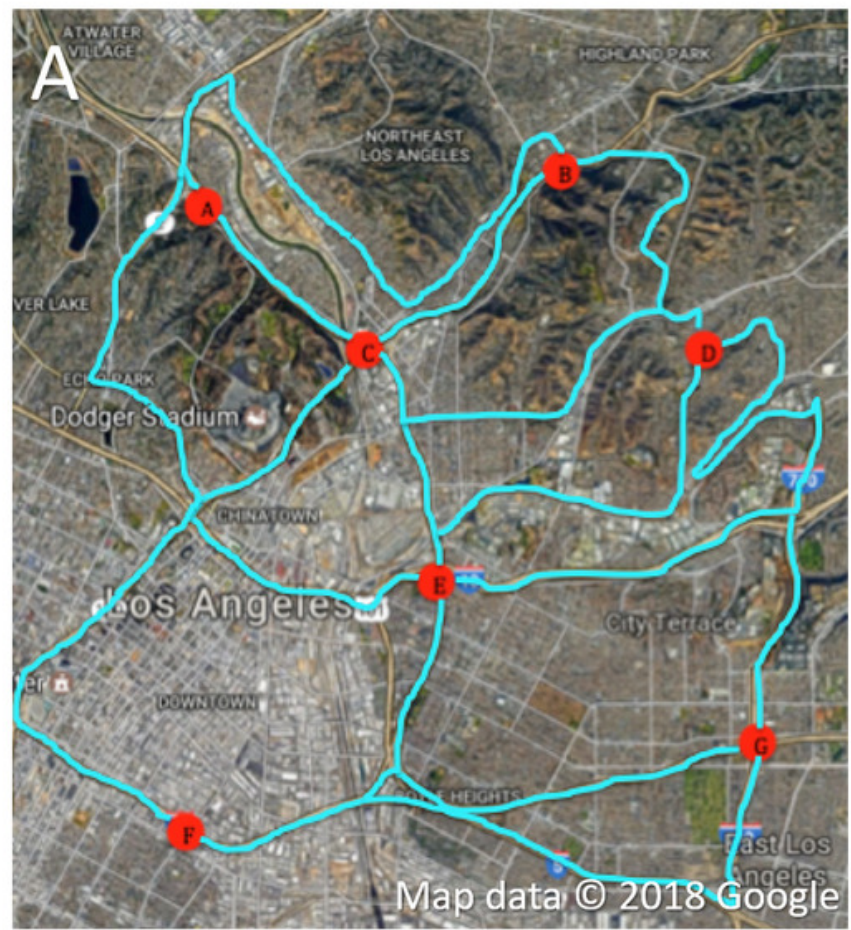

Slime Mold Exploration Networks

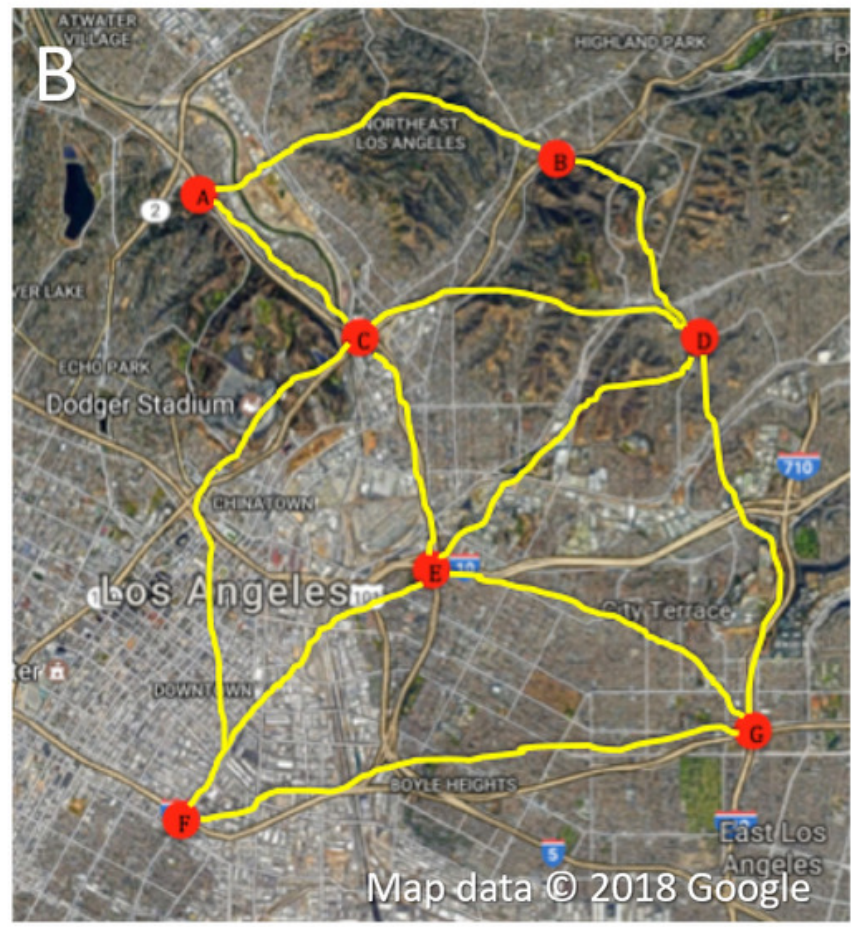




\section{Figure 4}

Calculated differences between slime mold and existing road network distances.

Difference in distance of the slime mold's entire exploration on 3D printed maps compared to that of existing highway networks.

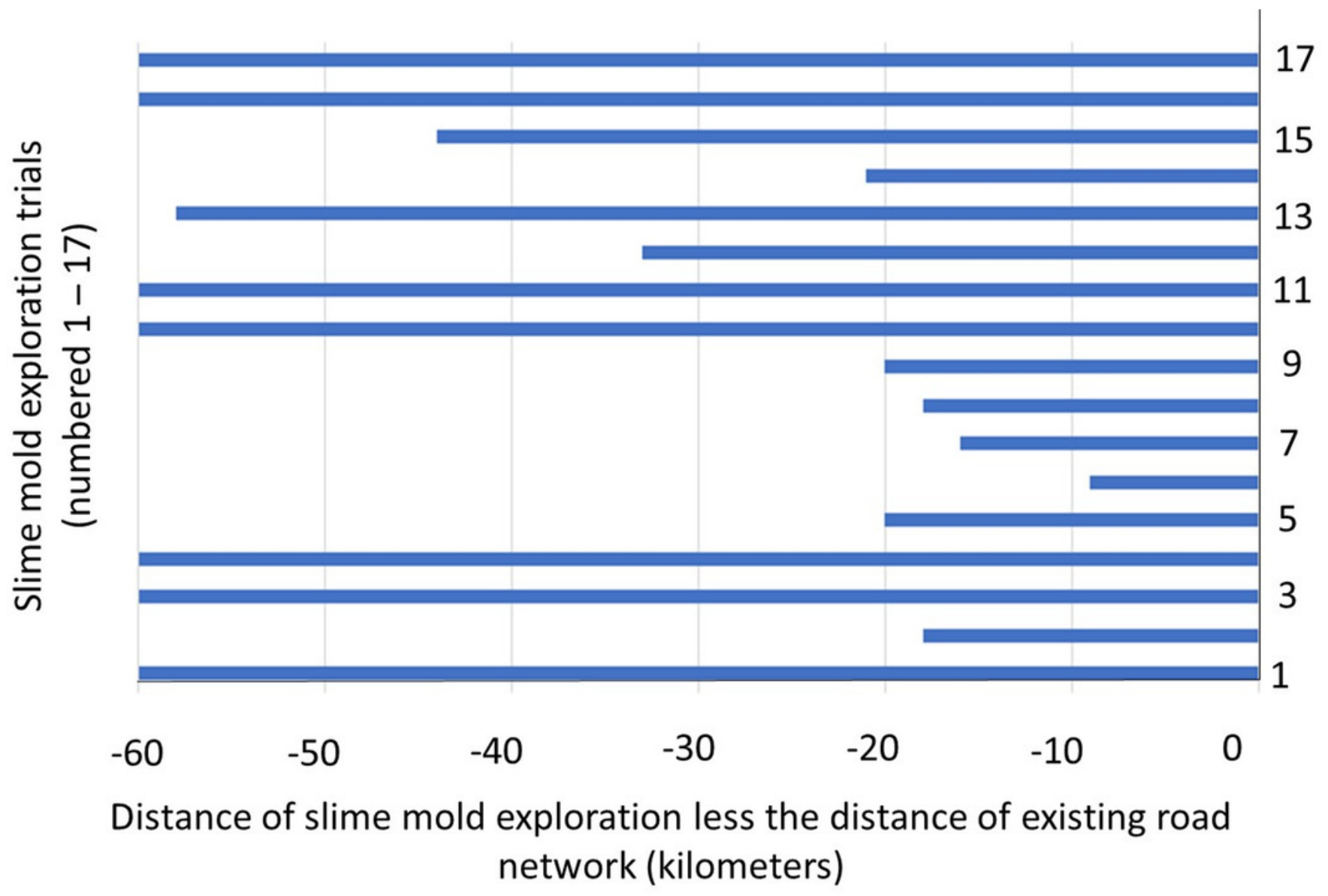

